\title{
INTEGRATION OF ACTIVITY-BASED MODELING AND DYNAMIC TRAFFIC ASSIGNMENT
}

\author{
Dung-Ying Lin ${ }^{*}$ \\ The University of Texas at Austin, \\ Department of Civil, Architectural \& Environmental Engineering \\ 1 University Station, C1761, Austin, TX 78712 \\ Phone: (512) 471-4539; Fax: (512) 475-8744; E-mail: dylin@mail.utexas.edu \\ *Corresponding Author
}

\section{Naveen Eluru}

The University of Texas at Austin, Department of Civil, Architectural \& Environmental Engineering 1 University Station, C1761, Austin, TX 78712

Phone: (512) 471-4535; Fax: (512) 475-8744; Email: naveeneluru@mail.utexas.edu

\section{S. Travis Waller}

The University of Texas at Austin, Department of Civil, Architectural \& Environmental Engineering 1 University Station, C1761, Austin, TX 78712

Phone: (512) 471-4539; Fax: (512) 475-8744; E-mail: stw@mail.utexas.edu

\section{Chandra R. Bhat}

The University of Texas at Austin, Department of Civil, Architectural \& Environmental Engineering 1 University Station, C1761, Austin, TX 78712

Phone: (512) 471-4535; Fax: (512) 475-8744; Email: bhat@mail.utexas.edu 


\begin{abstract}
The traditional trip based approach to transportation modeling has been employed for the past thirty years. However, due to the limitations of traditional planning for short-term policy analysis, researchers have explored alternative paradigms for incorporating more behavioral realism in planning methodologies. On the demand side, activity-based approaches have evolved as an alternative to traditional trip-based transportation demand forecasting. On the supply side, dynamic traffic assignment models have been developed as an alternative to static assignment procedures. Unfortunately, much of the research efforts in activity-based approaches (the demand side) and dynamic traffic assignment techniques (the supply side) have been undertaken relatively independently. To maximize benefits from these advanced methodologies, it is essential to combine them via a unified framework. The objective of the current paper is to develop a conceptual framework and explore practical integration issues for combining the two streams of research. Technical, computational and practical issues involved in this demand-supply integration problem are discussed. While the framework is general in nature, specific technical details related to the integration are explored by employing CEMDAP for activity-based modeling and VISTA for the dynamic traffic assignment modeling. Solution convergence properties of the integrated system, specifically examining different criteria for convergence, different methods of accommodating time of day and the influence of step size on the convergence are studied. Further, the integrated system developed is empirically applied to two sample networks selected from the Dallas Fort Worth network.
\end{abstract}




\section{INTRODUCTION}

For nearly thirty years, the traditional trip-based approach to transportation modeling has dominated the planning process. The trip-based method includes: trip generation, trip distribution, modal split and trip assignment. The first three steps of the trip-based method typically constitute the transportation demand side, while trip assignment normally represents the transportation supply side. Thus, the trip-based method accommodates transportation demand and supply within a somewhat unified framework when executed with full feedback. However, the trip-based approach is plagued with many limitations (for example, see (1), (2), (3), (4), (5) and (6)). This has led to an active stream of research that examines alternative paradigms for predicting travel demand and supply by incorporating more behaviorally realistic methodologies.

On the demand side, researchers have attempted to overcome the conceptual and behavioral inadequacy of the trip-based approach through the use of an activity-based modeling (ABM) paradigm. In this paradigm, it is recognized that travel is a derived demand and the need to travel arises from the more fundamental need to participate in activities. Activity-based approaches to modeling travel demand are conceptually more appealing compared to the trip-based method for the following reasons: (1) Treatment of time as a continuum and a generally superior incorporation of the temporal dimension, (2) Focus on sequences and patterns of activities and travel (i.e., tours) rather than individual trips, (3) Recognition of linkages among various activity-travel decisions, (4) Incorporation of intra-household interactions, inter-personal and intra-personal consistency measures, (5) Consideration of space-time constraints on activities and travel, and (6) Emphasis on individual level travel patterns. The potential benefits of the activity-based analysis and the resulting interest in operationalizing the activity-based approach have sparked an interest in micro-simulation based modeling systems. A number of micro-simulation platforms that employ the activity-based paradigm of transportation demand forecasting have been developed recently, such as CEMDAP [see (5) and (7)], Portland METRO [see (8)], New York NYMTC [see (9)], Columbus MORPC [see (10)], Sacramento SACOG [see (11)] and the San Francisco SFCTA [see (12)].

On the supply side, conventional techniques of trip assignment based on static traffic assignment (STA) have been employed for decades. The limitations of the static assignment procedures and the increase in computing capacity have allowed the field to move toward more behaviorally realistic dynamic traffic assignment (DTA) models. DTA techniques offer a number of advantages relative to the STA methods including: (1) Capturing time-dependent interactions of the travel demand and supply of the network, (2) Capability to capture traffic congestion build-up and dissipation, (3) Accommodating the affect of ramp-meters and traffic lights on the network are more straightforward, (4) Suited to model the effects of ITS technologies and (5) The network representation can be undertaken at a disaggregate level. A number of simulation-based DTA modules have been developed in the recent past such as VISTA [see (13)], CONTRAM [see (14)], DynaMIT [see (15-17)] and DYNASMART-P [see (18)].

It is evident that significant advancements have occurred on the demand and supply sides. However, the progress in the two streams has been achieved relatively 
independently. On the other hand, employing only one of these frameworks for travel demand modeling would yield inconsistent results and substantially fail to exploit the true potential of either approach. At a basic level, activity-based approaches typically consider time as a continuum, and predict activity-travel patterns in continuous-time. At the same time, DTA techniques are developed for the purpose of accommodating temporal dynamics of demand. Thus, using an ABM with a static assignment process that does not consider temporal dynamics undoes much of the advantages of predicting travel patterns in continuous-time. Similarly, using a trip-based approach that provides travel demands over an entire day or in 2-3 aggregate time periods of the day to develop the inputs for DTA does not exploit the very purpose for which DTA models have been developed. Therefore, to realize the benefits of these behaviorally realistic frameworks and obtain consistent results, it is imperative to develop a conceptually unified framework to draw from the advantages of research in either stream.

In this paper, we develop a conceptual framework for combining the progress made in the ABM and DTA areas of research, as well as explore the methodological, computational, and practical issues involved in integrating ABM demand systems with DTA-based supply systems.

The remainder of the paper is organized as follows. Section 2 reviews the research from earlier studies related to the current study. Section 3 proposes the fixed point formulation of the demand-supply integration problem. Section 4 describes the demand and supply system components and highlights the issues related to their integration. Section 5 presents empirical analysis undertaken with two sample networks. Section 6 concludes the paper.

\section{LITERATURE REVIEW}

The integration of transportation demand and supply has been of interest in recent years. Cantarella and Cascetta (19) discussed the theoretical results of the dynamic framework that processed the interaction between transportation demand and supply. Antoniou et al. (20) presented a pre-trip demand simulator that estimated dynamic O-D matrices. Lam and Huang (21) presented the mathematical formulations of both the time-dependent and dynamic activity choice to accurately represent the real time traffic conditions in dynamic or time-dependent traffic assignment. While pioneering, the two research efforts reviewed above did not use the feedback from DTA to update the input information for the demand simulator.

There have also been research efforts to address demand/supply integration by multi-agent simulation. Esser and Nagel (22) developed a multi-agent micro-simulation module that implemented the interaction among activity generation, route assignment and network loading. Raney et al. (23) developed an agent-based simulator that consisted of activity generation, modal and route choice, traffic simulation and learning/feedback modules. Raney and Nagel (24) proposed a model that included user routes generation, micro-simulation and feedback module that corrected the process. Rieser et al. (25) presented a model to couple activity-based demand generation with multi-agent traffic simulations. Though the integration of transportation demand and supply has been proposed for years, much of the research is still at the conceptual stage. 
Many transportation systems are based on some notion of equilibrium behavior, and thus can be formulated as variants of the basic fixed point problem. Cantarella (26) studied the multi-mode and multi-user equilibrium assignment with elastic demand and presented a fixed point formulation of the problem. Cascetta and Postorino (27) formulated the O-D count based estimation problem on congested network as a fixed point problem. Bar-Gera and Boyce (28) proposed a fixed point formulation of the consistent transportation forecasting models that combined static travel demand and network assignment. Estimation of O-D matrices from a partial set of traffic link volumes was studied in Sherali et al. (29). They proposed a fixed point formulation and introduced the nonlinear cost function. It was shown that the fixed point solution to the O-D matrices estimation from partial link volume information could be determined by successive linear programming approximation. Zhao and Kockelman (30) examined the existence and uniqueness of random-utility-based multi-regional input-output solution and formulated the problem as a fixed point problem.

A non-convex combined travel forecasting model was constructed by Bar-Gera and Boyce (31). Different step sizes in the method of successive averages for fixed-point problems were discussed in that work. Friesz and Mookherjee (32) investigated the infinite dimensional variational inequality formulation of dynamic user equilibrium (DUE) and differential variational inequality version of DUE. Martinez and Henriquez (33) investigated the static equilibrium in the real estate market and proposed a fixedpoint algorithm to solve the equilibrium.

This paper introduces the fixed point formulation of the integrated ABM and DTA when a variational inequality formulation of the dynamic user equilibrium traffic assignment is also incorporated in the model to capture user behavior. Following the formulation, a solution method is proposed to investigate the benefits of combining two behaviorally realistic frameworks.

\section{MATHEMATICAL FORMULATION}

Level-of-service (LOS) values are one of the critical inputs for the ABM system. The O$\mathrm{D}$ trip tables generated from the ABM system are loaded onto the network using DTA to obtain the LOS values. However, the LOS values obtained from DTA can be inconsistent with the LOS values used in the ABM system. Ideally, the assignment of trip tables onto the network should result in the "same" LOS values used in finding the trip tables. This consistency can be achieved by the iterating of the integrated ABM and DTA. To this end, we formulate the problem as a fixed point problem and propose an iterative algorithm in later sections. We first introduce the following notation:

$$
\begin{array}{ll}
\Xi & =\text { vector that represents any feasible DTA } \\
\Xi^{*} & =\text { vector that represents the "optimal” DTA } \\
\Psi(\Xi) & =\text { vector that represents the path cost resulting from the DTA } \Xi \\
Z(\Psi(\Xi)) & =\text { dynamic trip table resulting from the path cost vector } \Psi(\Xi) \\
P(Z(\Psi(\Xi))) & =\text { user paths vector from assigning trip tables } Z(\Psi(\Xi))
\end{array}
$$




$$
\begin{array}{ll}
S(P(Z(\Psi(\Xi)))) & =\text { path cost vector obtained from simulating user } \\
& \text { paths } P(Z(\Psi(\Xi)))
\end{array}
$$

The integration of ABM and DTA can be formulated as equation (3.1) and (3.2).

$$
\begin{array}{ll}
\Psi\left(\Xi^{*}\right)^{T}\left(\Xi-\Xi^{*}\right) \geq 0 & \forall \Xi \in D \\
\Psi(\Xi)=S(P(Z(\Psi(\Xi)))) & \forall \Xi \in D
\end{array}
$$

Equation (3.1) is a variational inequality (VI) formulation of the Wardrop-type dynamic user equilibrium traffic assignment (Chang, see (34)). It can be observed that the user equilibrium DTA $\Xi^{*}$ always results in lower total route cost than other feasible assignments by rearranging equation (3.1) to $\Psi\left(\Xi^{*}\right)^{T} \Xi \geq \Psi\left(\Xi^{*}\right)^{T} \Xi^{*}$.

Equation (3.2) is the fixed point formulation of the interaction between ABM and DTA. Function $Z$ corresponds to the ABM system. It takes the LOS values as its input and outputs the O-D trip tables after the function evaluation. Function $P$ and $S$ correspond to the path-finding module and simulation module respectively in DTA. We input the O-D trip tables into function $P$ and it determines the time-dependent user paths. Function $S$ then simulates those paths and obtains the LOS values $\Psi(\Xi)$. Ideally, the function evaluation with input vector $\Psi(\Xi)$ on the right-hand-side of equation (3.2) should give the identical $\Psi(\Xi)$ on the left-hand-side of the equation. The fixed point formulation with the VI constraint can be solved in an iterative manner.

\section{SYSTEM}

In this section, the integrated framework is introduced. First the two primary components of the framework CEMDAP (ABM module) and VISTA (DTA module) will be overviewed. Integration issues will then be discussed.

\subsection{CEMDAP Framework}

The Comprehensive Econometric Micro-simulator for Daily Activity-travel Patterns (CEMDAP) is a micro-simulation implementation of a continuous-time activity-travel modeling system. CEMDAP takes as input information on the aggregate socioeconomics and the activity-travel environment characteristics in the urban study region for the base year, as well as policy actions being considered for future years (the activity-travel environment includes the land-use, urban form, and transportation LOS characteristics). The aggregate-level base year socioeconomic data are first fed into the synthetic population generator (SPG) to produce a disaggregate-level synthetic dataset describing a subset of the socioeconomic characteristics of the households and individuals residing in the study area (see (35) for information on the SPG module). Additional base-year socioeconomic attributes related to mobility, schooling, and employment at the individual level, and residential/vehicle ownership choices at the household level, that are difficult to synthesize (or cannot be synthesized) directly from the aggregate socioeconomic data for the base year are simulated by the Comprehensive Econometric Microsimulator for SocioEconomics, Land-use, and Transportation System (CEMSELTS), (see (36) for more 
details). The base year socioeconomic data, along with the activity-travel environment attributes, are then run through the CEMDAP to obtain individual-level activity-travel patterns (see (5) and (7) for details). The activity-travel patterns are subsequently passed through a dynamic traffic micro-assignment scheme to determine path flows, link flows, and transportation system LOS by time of day. In the framework, the initial iteration of CEMDAP needs the LOS values as inputs. However, the values used in the iteration need not be the "true" LOS values. So it is necessary to rerun the CEMDAP module with the new LOS variables obtained.

\subsection{VISTA Framework}

Visual Interactive System for Transport Algorithms (VISTA) is a comprehensive DTA system that integrates data warehousing and traffic analysis for transport applications via a client-server implementation. VISTA was originally outlined in Waller and Ziliaskopoulos (13). As with many contemporary simulation-based DTA approaches, VISTA is comprised of three primary modules: traffic simulation, time-dependent routing algorithms, and path assignment.

The traffic simulator in VISTA is RouteSim [see (37)], a route-based traffic simulator based on the Cell Transmission Model [see (38-39)]. RouteSim takes a network (nodes, links and controls) as well as the spatial path assignment as input and outputs the spatio-temporal trajectories of travelers. The time-dependent shortest path (TDSP) module is implemented according to Ziliaskopoulos and Mahmassani [see (40, 41)] and has substantial potential for distributed and parallel implementations (Ziliaskopoulos and Kotzinos, (42)) which is critical for large-scale deployments.

Path assignment in VISTA is handled through multiple means. The traditional MSA approach is employed for early iterations, but gap function based methods are employed to obtain meaningful convergence in later iterations. For the latter a variety of gap functions are employed which are based on the variational inequality formulation as detailed in Chang (34).

VISTA typically employs time-scales of approximately 6 seconds for traffic dynamics (for simulation, time-dependent routing, and trip departure times). A scale of approximately 5 minutes is common for path choice behavior (i.e., travelers departing within 5 minutes of each other between the same origin-destination pair will observe similar conditions). It should be noted that this minor 5-minute aggregation occurs after TDSPs have been found based on the 6 second scale.

The path assignment and TDSP modules were reengineered into an efficient module that can handle large data sets in Ziliaskopoulos and Waller (43). Ziliaskopoulos et al. (44) developed an Internet-based geographic information system (GIS) and incorporated it into the system framework. This equipped VISTA with the unique feature of being accessed over the Internet via web browser, CORBA interface or Java GIS. The feature eliminates the need for software installation/upgrade and allows users to conveniently access the consistent analysis without spatial limitation.

\subsection{Integration}


The integration of CEMDAP and VISTA poses methodological and technical challenges. In the current section, we discuss how these challenges are addressed in the proposed approach.

The ABM requires the LOS values (primarily travel time) as inputs to generate activity travel patterns. However, it is possible that these input values do not correspond to the actual travel times. Therefore, the activity patterns generated need to be translated into O-D matrices by time of day and loaded onto the network (through the DTA model) to produce the travel times. This clearly highlights the necessity of an iterative procedure between the ABM and the DTA model. An important consideration here would be to determine the convergence criterion to stop the iterations. In the integrated model we generate trip tables that form the input to obtain the travel times and vice versa. After every iteration, O-D matrices of the current and the previous iteration can be compared. Similarly travel time from the current and previous iterations can be compared. Potentially, two measures of convergence exist: (1) Trip table convergence and (2) Travel time convergence. The convergence criterion is based on the attribute that is averaged after the iteration (with MSA techniques) and the attribute that needs to converge (across iterations). In trip table convergence, travel time values are averaged after the iteration and trip table convergence is then checked, while in the trip table convergence, travel time and trip tables are used in the opposite roles. If the average of difference is less than predefined stopping criterion, we stop the integration and treat the results as the converged solution. To be specific, the equations employed to measure the convergence are outlined below:

Let TT denotes the travel time, $k$ denotes the current number of iterations, $N$ denotes the total number of O-D pairs and NT denotes the number of trips. We define:

Average Travel Time for O-D $=\frac{1}{k} \times T T_{o d}^{k}+\frac{k-1}{k} \times T T_{o d}^{k-1}$

Average Difference of Trip (\%) $=\frac{1}{N} \times \sum_{o d \in O D} \frac{\left|N T_{o d}^{k-1}-N T_{o d}^{k}\right|}{N T_{o d}^{k}} \times 100$

Average Trip for O-D $=\frac{1}{k} \times N T_{o d}^{k}+\frac{k-1}{k} \times N T_{o d}^{k-1}$

Average Difference of Travel Time (\%) $=\frac{1}{N} \times \sum_{o d \in O D} \frac{\left|T T_{o d}^{k-1}-T T_{o d}^{k}\right|}{T T_{o d}^{k}} \times 100$

Based on the definitions of convergence described earlier, if we average travel times between O-D pairs (equation (4.1)), we employ difference of trips (equation (4.2)) as the convergence criterion. If we average O-D trips (equation (4.3)) after the iteration, we use average difference in travel time (equation (4.4)) as the convergence criterion. The final framework developed for the integration is presented in FIGURE 1. The framework suits the application of both methods of convergence for integration of CEMDAP and VISTA.

It should be noted here that although MSA is one of the most practical measures and drives the solution towards convergence, it is not without its share of limitations. For instance, individual behaviors are averaged during the process and it does not guarantee convergence to the right point. However, an important aspect of this research is to develop a conceptual structure and to find a pragmatic approach. Advanced approaches such as gap function based methods should be developed for convergence and realistic behaviors in the future. 
In addition to the conceptual challenges, we must address technical issues related to integration. For the trip table convergence procedure, CEMDAP generates activity travel patterns in continuous time. These activity patterns need to be converted into dynamic O-D matrices. These matrices are provided to the VISTA framework to load the network with these trips. Within VISTA, the network assignment undertakes traffic simulation, optimal routing and path assignment to obtain the traffic link volumes and speeds. The travel times obtained from VISTA are appropriately processed and provided as input to CEMDAP (travel times are provided by time of day in CEMDAP). With these new inputs CEMDAP generates new activity travel patterns. These are again converted into $\mathrm{O}-\mathrm{D}$ trip tables by time of day. At this juncture, we check if the O-D trip tables generated in the current iteration are close to the O-D trip values generated in the last iteration. If the O-D matrices converge, the process is terminated and the O-D trip tables with the corresponding link volumes and speeds are provided for analysis. If the O-D matrices have not converged, the iteration continues. The procedure highlighted, is very similar for the travel time convergence methodology.

One more technical challenge is the communication between the CEMDAP and VISTA models. CEMDAP is designed to work on a Windows platform while VISTA is designed to work on a Linux platform. To effectively address this, the two modules installed on two separate machines are connected via a local area network. The activity travel patterns generated from CEMDAP are converted to the dynamic O-D matrices, copied to Linux machine, and uploaded to VISTA's PostgreSQL database. The time of day LOS values generated from VISTA are copied to Windows machine and uploaded to CEMDAP's PostgreSQL database. The copy and uploading are implemented in Java programming language using Secure Shell protocol (SSH).

\section{NUMERICAL EXPERIMENTS}

The proposed integration is tested on two sample networks. The demographic information required for CEMDAP is obtained by sampling the Dallas Fort Worth demographic data generated using SPG and CEMSELTS (refer to (36) for more details). The network data essential for VISTA are obtained by sampling data sets provided by North Central Texas Council of Government. The running environment of VISTA is Linux with an Intel $3.00 \mathrm{GHz} \mathrm{CPU}$ and $32 \mathrm{~GB}$ memory; while the environment of CEMDAP is Windows XP with Intel $3.4 \mathrm{GHz}$ CPU and 2 GB memory.

In the experiments, we employ two measures of convergence presented in previous section: (1) Trip table convergence and (2) Travel time convergence. In addition to the convergence criterion we also adopt a maximum number of iterations based on the computational burden. Further, in the current analysis we employ two different partitions of time of day for the purpose of the empirical analysis. In the first category the entire day is treated as a single partition. In the second category we split the day into five periods - AM peak (6:30-9:00), PM peak (16:00-18:30) and off peak periods (0:00-6:30, 9:00-16:00 and 18:30-24). It is evident that in the first category we do not differentiate between peak and off peak periods. The travel times and trips obtained are averaged over each time period. The empirical results are presented in the following subsections.

\subsection{Grid Network}


The grid network presented in FIGURE 2 composes of 69 nodes/zones and 218 links. The total number of O-D pairs is $4,761(69 \times 69)$. The maximum iteration is set to 200 for the experiments on this network. On average, it takes about five hours to complete 200 iterations for this network. As the number of households, size of the network and total number of O-D pairs are relatively small, we set a strict convergence criterion for the analysis (convergence criterion is set to $0.0001 \%$ ). We conduct five experiments with this network. The results are summarized in FIGURE 3 and FIGURE 4 respectively.

\subsubsection{Experiment 1: Single Time-interval and Trip Table Convergence}

For the first experiment, a single time-interval partition and trip table convergence are employed. The computational results are summarized in the first chart of FIGURE 3. The test converges after 140 iterations. The average differences of both the trip table and travel time are $0 \%$ in the end. It can be observed from the figure that the average difference in the trip table falls rapidly in the initial stages of the iteration. After the $10^{\text {th }}$ iteration the average difference of trips remains below 5\% until convergence. Therefore, potentially choosing $5 \%$ of the average difference as the stopping criterion might be effective in practice.

\subsubsection{Experiment 2: Single Time-interval and Travel Time Convergence}

In the second test we employ a single time interval and travel time convergence. The results are summarized in the second chart in FIGURE 3.

The test converges after iteration 76; the average difference of the trip table is $1.50 \%$ and the difference of travel time reaches $0 \%$ in the end. The average trip difference decreases monotonously since we use the MSA-type of equation (4.3) to average trips. However, as can be seen from FIGURE 3, the average difference of travel time fluctuates significantly during the iterative process. The average travel time difference does not drops below 5\% until iteration 47. Even after that, the difference oscillates in later iterations. In this experimental setup, it would not be beneficial to adopt a relaxed convergence criterion to achieve convergence earlier. It should be noted that if the convergent values were slightly perturbed at iteration 76 , the iteration may start oscillating again. This brings up a broader issue about convergence. Arriving at a stricter definition of convergence might allow us to address this issue. Currently, averaging travel time and trip table convergence in the first experiment is the appropriate convergence criterion.

\subsubsection{Experiment 3: Multiple time-intervals and Trip Table Convergence}

In the subsequent test, we employ multiple time-intervals and a trip table convergence criterion. This test converges at iteration 64 (see the third chart of FIGURE 3 ) while the average difference of both travel time and trips reach $0 \%$. Compared to the corresponding single time-interval case, the multiple time-intervals test converges faster; both in terms of CPU time and number of iterations (64 versus 140). This is expected because partitioning the day allows for more accurate predictions of travel time thereby leading to smaller differences across iterations. In a single time period case, peak and off-peak travel are averaged to arrive at the average time for the entire period. However, 
in the multiple time-intervals case different averages for different intervals are evaluated leading to better estimates of travel time.

\subsubsection{Experiment 4: Multiple Time-intervals and Travel Time Convergence}

In the next test, we still consider multiple time-interval case. However, we use travel time convergence. The test converges at iteration 34; the average difference of trip is $1.50 \%$ at the last iteration (see the fourth chart of FIGURE 3). The difference of travel time reaches $0 \%$ in the end. It can be observed that the fluctuation of average travel time difference is slightly larger than the fluctuation in Experiment 3. However, fewer iterations are required for this process to converge. Also, comparing with Experiment 2, the multiple time-interval case still converges faster than the single time-interval case. The same reasoning provided for the previous test applies here.

From the experiments conducted we can make the following observations: (1) Trip table convergence results in a rapid fall in the trip table difference in the first few iterations, (2) Travel time convergence results in a slower initial drop in travel time difference but reaches final convergence in fewer iterations, (3) Partitioning the day into multiple time periods improves convergence for both means of convergence. Based on these observations we employ multiple time intervals and trip table convergence for all subsequent tests.

\subsubsection{Experiment 5: Step-Size Tests}

In the following experiments, we examine the significance of step-sizes in convergence. Instead of using the step size $\frac{1}{k}$ in Equation (4.1), we use the fixed step size 1, 5, 10 and 20 respectively. The results are depicted in FIGURE 4.

It can be seen from FIGURE 4, when the step size is small (1, 5 and 10), the integration does not converge before the maximum iteration is reached. However, it converges when step size is increased to 20. The test with step size of 20 converges at iteration 129 with $0.01 \%$ average difference of travel time and $0 \%$ average difference of trips. The crucial role of step size in the convergence can be observed from these results. It is evident that only suitably chosen step sizes can lead to better convergence in the integrated modeling of transportation demand and transportation LOS measures. Also, from the figure it can be observed that large step size results in larger fluctuation of average trip table difference.

\subsection{Ring Roadway Network}

The second sample network FIGURE 5 is a grid network surrounded by a ring roadway system. The network composes of 358 nodes/zones and 650 links. Only the households and trips generated within the network are considered in the numerical experiment. To obtain the results within reasonable computational time, the maximum iteration is set to 15 in this experiment.

The CPU time required for 15 full-feedback iterations on this ring roadway network is 85 hours. It should be noted that, both components of the framework have the capability for substantially scalable distribution. However, distributed capabilities were 
not employed for these tests to ease the technical aspects of integration. The convergence of the ring roadway network test is presented in FIGURE 6.

The average travel time difference is $319.96 \%$ at the first iteration and decreases to $0.40 \%$ at iteration 15 . The average trip difference begins from $76.88 \%$ and decreases to $3.75 \%$ at the end of the test. The difference in trip table values reduces rapidly and reaches an acceptable value within 15 iterations. Also, the difference across iteration is monotonously reducing and relatively stable.

\section{CONCLUSION}

The traditional trip-based approach to transportation modeling is plagued with numerous limitations. This paper examines an alternative solution of predicting travel demand and supply by incorporating more behaviorally realistic methodologies. The problem is formulated as a fixed point problem with a VI DTA constraint (equation (3.2)). On the demand side, we employ ABM instead of trip-based approach for travel demand forecasting. On the supply side, we apply behaviorally more realistic DTA instead of static traffic assignment. In this paper, CEMDAP serves as the tool in the demand side analysis while VISTA is the tool for supply side analysis. An integrated system is developed for combining the progress made in the two streams of research in a unified framework. The technical, computational and practical issues involved in this demandsupply integration problem have been extensively discussed.

In particular, the paper examines the convergence properties of the unified tool towards modeling transportation demand and transportation LOS measures. In the empirical application, we examine different criterion for convergence, different means of partitioning the day and influence of step size on the convergence. From the results, it is evident that trip table convergence criterion, multiple time interval portioning and varying step size yield faster and more stable convergence results.

\section{ACKNOWLEDGEMENTS}

This material is based in part upon work supported by the National Science Foundation under Grant Number CMMI-0347005. Any opinions, findings, and conclusions or recommendations expressed in this material are those of the author(s) and do not necessarily reflect the views of the National Science Foundation. 


\section{REFERENCE}

1. Jones, P. M., Koppelman, F.S. and Orfeuil, J.P. “Activity Analysis: State of the Art and Future Directions" In Developments in Dynamic and Activity-Based Approaches to Travel Analysis, pp. 34-55, Gower, Aldershot, England, 1993.

2. Axhausen, K. and Gärling, T. “Activity-based Approaches to Travel Analysis: Conceptual Frameworks, Models and Research Problems” Transport Reviews, 12, pp. 324-341, 1992.

3. Kurani, K.S. and Kitamura, R. "Recent developments and the prospects for modeling household activity schedules” Report prepared for the Los Alamos National

Laboratory, Institute of Transportation Studies, University of California, Davis, CA, 1996.

4. Bhat, C.R. and Koppelman F. Activity-Based Modeling for Travel Demand. Handbook of Transportation Science, R.W. Hall (ed.), Kluwer Academic Publisher, 1999.

5. Bhat, C.R., Guo, J.Y., Srinivasan, S. and Sivakumar, A. "A Comprehensive Econometric Microsimulator for Daily Activity-Travel Patterns” Transportation Research Record, Vol. 1894, pp. 57-66, 2004.

6. Vovsha, P. and Bradley, M. “Advanced Activity-Based Models in a Context of Planning Decisions” Transportation Research Record 1981, Journal of the Transportation Research Board, TRB, National Research Council, Washington, D.C., pp. 34-41, 2005.

7. Pinjari, A., Eluru, N., Copperman, R., Sener, I.N., Guo, J.Y., Srinivasan, S. and Bhat, C.R. "Activity-Based Travel-Demand Analysis for Metropolitan Areas in Texas: CEMDAP Models, Framework, Software Architecture and Application Results” Report 4080-8, prepared for the Texas Department of Transportation, 2006.

8. Bowman, J. L., Bradley, M. A., Shiftan, Y., Lawton, T. K. and Ben-Akiva, M. E. "Demonstration of an Activity Based Model System for Portland", 8th World Conference on Transport Research, July 12-17, Antwerp, Belgium, 1998.

9. Vovsha P., Petersen, E. and Donnelly, R. "Micro-Simulation in Travel Demand Modeling: Lessons Learned from the New York Best Practice Model” Transportation Research Record 1805, pp. 68-77, 2002.

10. Vovsha, P., Petersen, E. and Donnelly R. "Impact of Intra-Household Interactions on Individual Daily Activity-Travel Patterns” Presented at the 83rd Annual Meeting of the Transportation Research Board, Washington, D.C., 2004.

11. Bowman, J. L., and Bradley, M. A. "Activity-Based Travel Forecasting Model for SACOG: Technical Memos Numbers 1-11” 2005-2006, Available at http://jbowman.net. 
12. Bradley, M., Outwater, M., Jonnalagadda, N., and Ruiter, E. "Estimation of an Activity-Based Micro-Simulation Model for San Francisco”. Presented at the 80th Annual Meeting of the Transportation Research Board, Washington D.C., 2001.

13. Waller, S.T. and Ziliaskopoulos, A.K. "A Visual Interactive System for Transportation Algorithms" Presented at the 78th Annual Meeting of the Transportation Research Board, Washington, D.C, 1998.

14. Taylor, N. B. “CONTRAM 5: an Enhanced Traffic Assignment Model” TRLL Research Report 249, 1990.

15. Ben-Akiva, M., Bierlaire, M., Koutsopoulos, H. and Mishalani, R. "DynaMIT: a Simulation-based System for Traffic Prediction and Guidance Generation” Paper presented at TRISTAN III, 1998.

16. Ben-Akiva, M., Bierlaire, M., Koutsopoulos, H. and Mishalani, R. "DynaMIT: a Simulation-based System for Traffic Prediction" Paper presented at the DACCORD Short Term Forecasting Workshop, 1998.

17. Massachusetts Institute of Technology "DTA System Enhancement and Evaluation at Traffic Management Center, Task P: Framework for the Use of DynaMIT 145 in Transportation Planning Applications (DynaMIT-P)” Technical report, Submitted to Oak Ridge National Laboratories, 2000.

18. Mahmassani, H. S., Hu, T-Y. and Jayakrishnan, R. "Dynamic Traffic Assignment and Simulation for Advanced Network Informatics (DYNASMART)” In Proceedings of the 2nd International CAPRI Seminar on Urban Traffic Networks, Capri, Italy, 1992.

19. Cantarella, G.E. and Cascetta, E. "Dynamic Processes and Equilibrium in Transportation Networks: Towards a Unifying Theory” Transportation Science Vol. 29, No. 4, pp. 305-329, 1995.

20. Antoniou, C., Ben-Akiva, M., Bierlaire, M. Mishalani, R "Demand Simulation For Dynamic Traffic Assignment" Presented at the 8th IFAC Symposium on Transportation Systems, Chania, Greece, 1997.

21. Lam, W.H.K and Huang, H.J “Combined Activity/Travel Choice Models: TimeDependent and Dynamic Versions” Networks and Spatial Economics, 3, pp. 323-347, 2003.

22. Esser, J. and Nagel, K. "Iterative Demand Generation for Transportation Simulations" The Leading Edge of Travel Behavior Research, pp. 659-681, 2001.

23. Raney, B., Cetin, N., Vollmy, A., Vrtic, M., Axhausen, K. and Nagel, K “An AgentBased Microsimulation Model of Swiss Travel: First Results” Networks and Spatial Economics, 3, pp. 23-41, 2003.

24. Raney, B. and Nagel, K. "Iterative Route Planning for Large-scale Modular 
Transportation Simulations” Future Generation Computer Systems, 20, pp.11011118, 2004

25. Rieser, M., Nagel, K., Beuck, U., Balmer, M., Rümenapp, J. “Truly Agent-oriented Coupling of an Activity-based Demand Generation with a Multi-agent Traffic Simulation” Transportation Research Board 2007 Annual Meeting CD-ROM

26. Cantarella, G.E. “A General Fixed-Point Approach to Multimode Multi-User Equilibrium Assignment with Elastic Demand” Transportation Science Vol. 31, No. 2, pp. 107-128, 1997

27. Cascetta, E. and Postorino, M.N. "Fixed Point Approaches to the Estimation of O/D Matrices Using Traffic Counts on Congested Networks” Transportation Science Vol. 35, No. 2, pp. 134-147, 2001.

28. Bar-Gera, H. and Boyce, D. “Origin-based Algorithms for Combined Travel Forecasting Models” Transportation Research Part B: Methodology 37, pp. 405-422, 2003.

29. Sherali, H.D., Narayanan, A. and Sivanandan, R.” Estimation of Origin-destination Trip-tables Based on a Partial Set of Traffic Link Volumes” Transportation Research Part B: Methodology 37, pp. 815-836, 2003.

30. Zhao, Y. and Kockelman, K.M. “The Random-utility-based Multiregional Inputoutput Model: Solution Existence and Uniqueness” Transportation Research Part B: Methodology 38, pp.789-807, 2004.

31. Bar-Gera, H. and Boyce, D. "Solving a Non-convex Combined Travel Forecasting Model by the Method of Successive Averages with Constant Step Sizes" Transportation Research Part B: Methodology 40, pp. 351-367, 2006.

32. Friesz, T.L. and Mookherjee, R. "Solving the Dynamic Network User Equilibrium Problem with State-dependent Time Shifts" Transportation Research Part B: Methodology 40, pp.207-229, 2006.

33. Martinez, F. J. and Henriquez, R. "A Random Bidding and Supply Land Use Equilibrium Model” Transportation Research Part B: Methodology 41, pp.632-651, 2007.

34. Chang, E. J. “Time-Varying Intermodal Person Trip Assignment” Ph.D. Dissertation, Northwestern University, 2004.

35. Guo, J.Y., and Bhat, C.R. "Population Synthesis for Microsimulating Travel Behavior” Forthcoming, Transportation Research Record, 2006.

36. Eluru, N., Pinjari, A.R., Guo, J.Y., Sener, I., Srinivasan, S., Copperman, R. and Bhat, C.R. "Population Updating System Structures And Models Embedded Within The Comprehensive Econometric Microsimulator For Urban Systems (Cemus)” Technical 
Paper, Department of Civil, Environmental and Architectural Engineering, The University of Texas at Austin, 2007.

37. Ziliaskopoulos, A. K., and Lee, S. “A Cell Transmission Based Assignmentsimulation Model for Integrated Freeway/surface Street Systems” Presented at the 75th Annual Meeting of the Transportation Research Board, Washington, D.C, 1996.

38. Daganzo, C. F. “The Cell Transmission Model: A Simple Dynamic Representation of Highway Traffic Consistent with the Hydrodynamic Theory” Transportation Research Part B: Methodology 28B, pp. 269-287, 1994.

39. Daganzo, C. F. "The Cell Transmission Model, Part II: Network Traffic" Transportation Research Part B: Methodology 29B, pp.79-93, 1995.

40. Ziliaskopoulos, A.K. and Mahmassani, H.S. "A Time Dependent Shortest Path Algorithm for Real-time Intelligent Vehicle/highway Systems" Transportation Research Record 1408, pp.94-104, 1993.

41. Ziliaskopoulos, A.K. and Mahmassani, H.S. "A Note on Least Time Path Computations Considering Delays and Prohibitions for Intersection Movements" Transportation Research Part B, 30(5), pp. 359-367, 1996.

42. Ziliaskopoulos, A.K. and Kotzinos, D. “A Massively Parallel Time-dependent Leasttime-path Algorithm for Intelligent Transportation Systems Applications” Comput. Aided Civ. Infrastruct. Eng. 16 (5) (2001) 337 -346 (Special Issue: High Performance Computing

43. Ziliaskopoulos, A.K., Waller, S.T., Li, Y. and Byram, M. "Large Scale Dynamic Traffic Assignment: Implementation Issues and Computational Analysis," Journal of Transportation Engineering Volume 130, No. 5, pp. 585-593, 2004.

44. Ziliaskopoulos, A.K. and Waller, S.T. "An Internet Based Geographic Information System that Integrates Data, Models, and Users for Transportation Applications" Transportation Research Part C Vol. 8 pp. 427-444, 2000. 


\section{LIST OF FIGURE CAPTIONS}

FIGURE 1: Integrated System

FIGURE 2: Grid Network

FIGURE 3: Convergence with Different Criteria

FIGURE 4: Convergence with Different Step Sizes

FIGURE 5: Ring Roadway Network

FIGURE 6: Multiple Time-intervals and Trip Table Convergence 


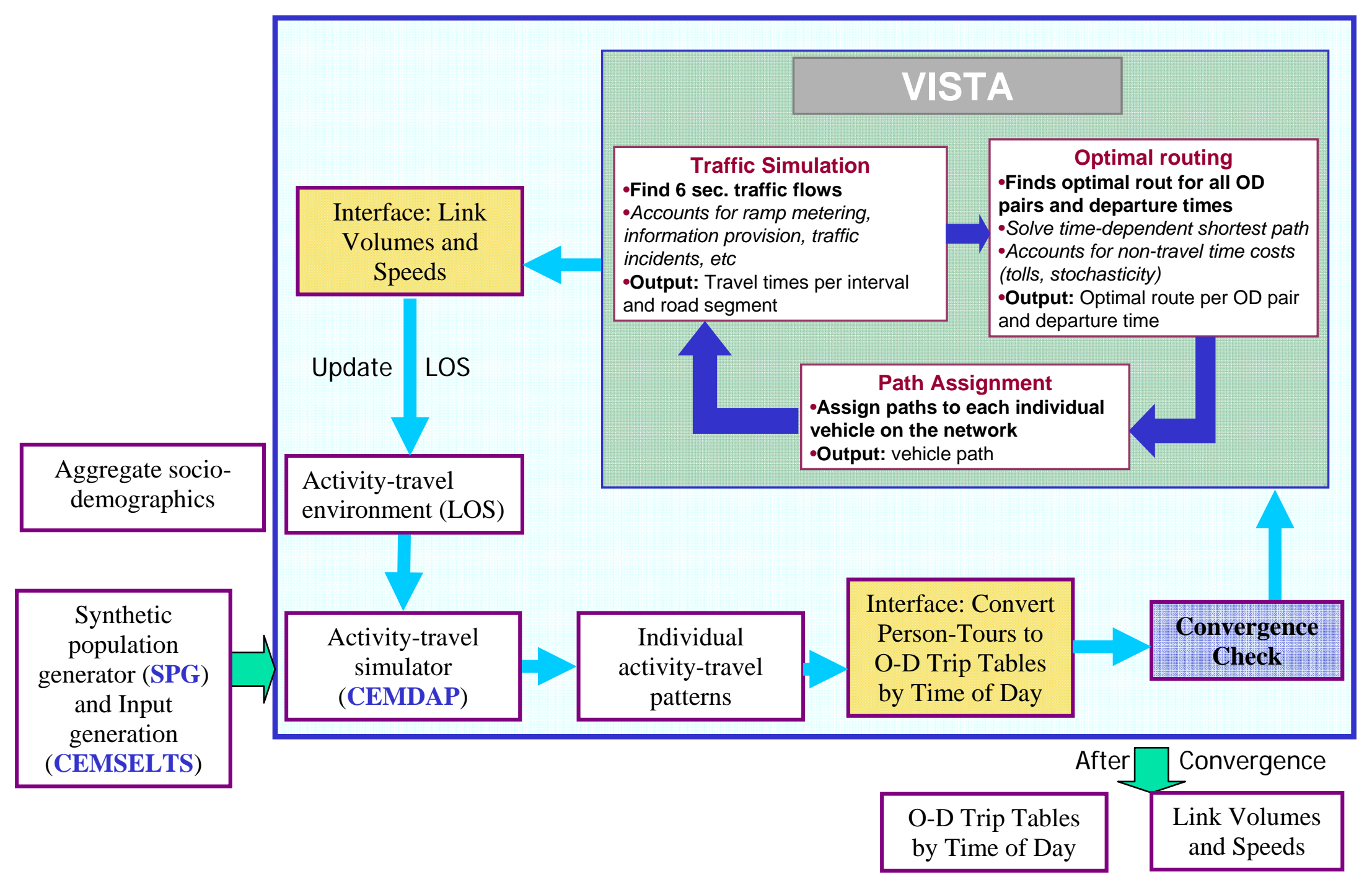

FIGURE 1: Integrated System 


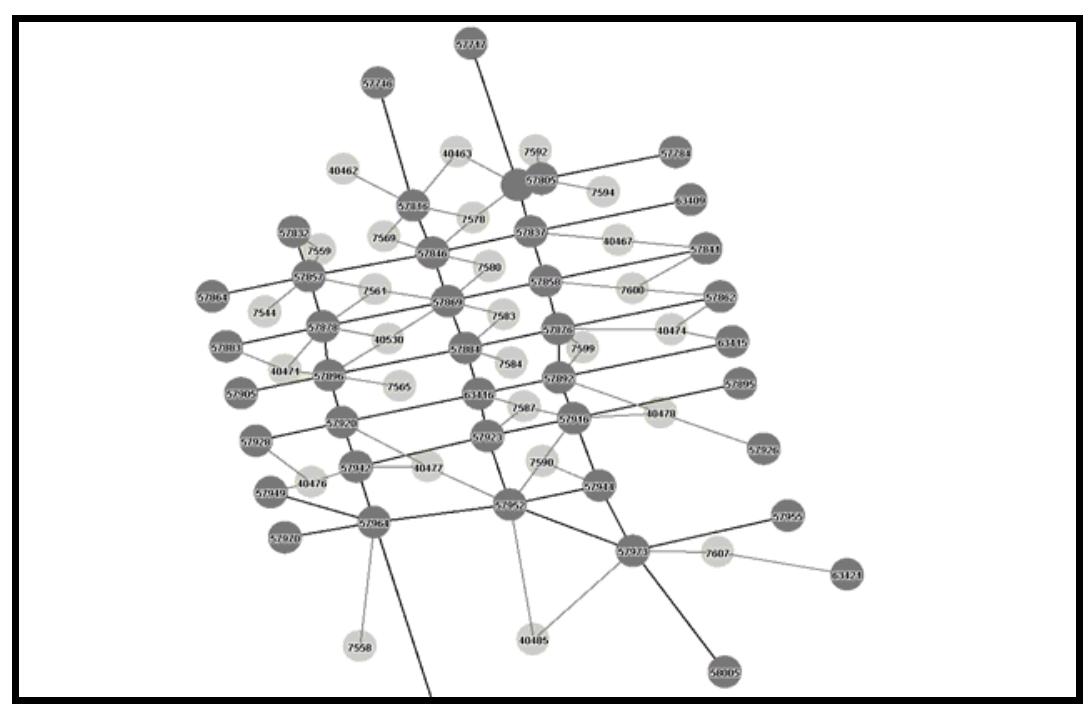

FIGURE 2: Grid Network 

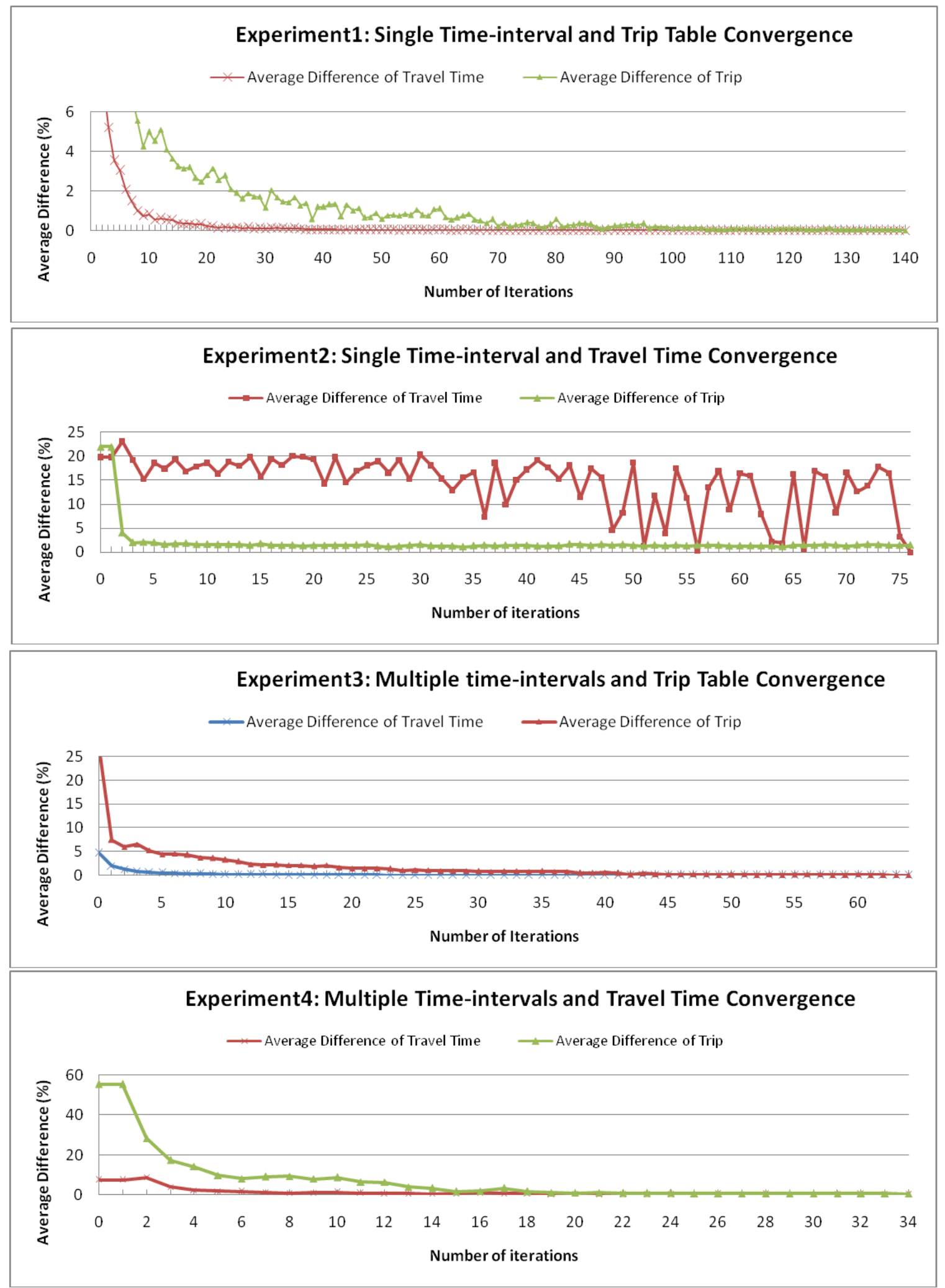

FIGURE 3: Convergence with Different Criteria 


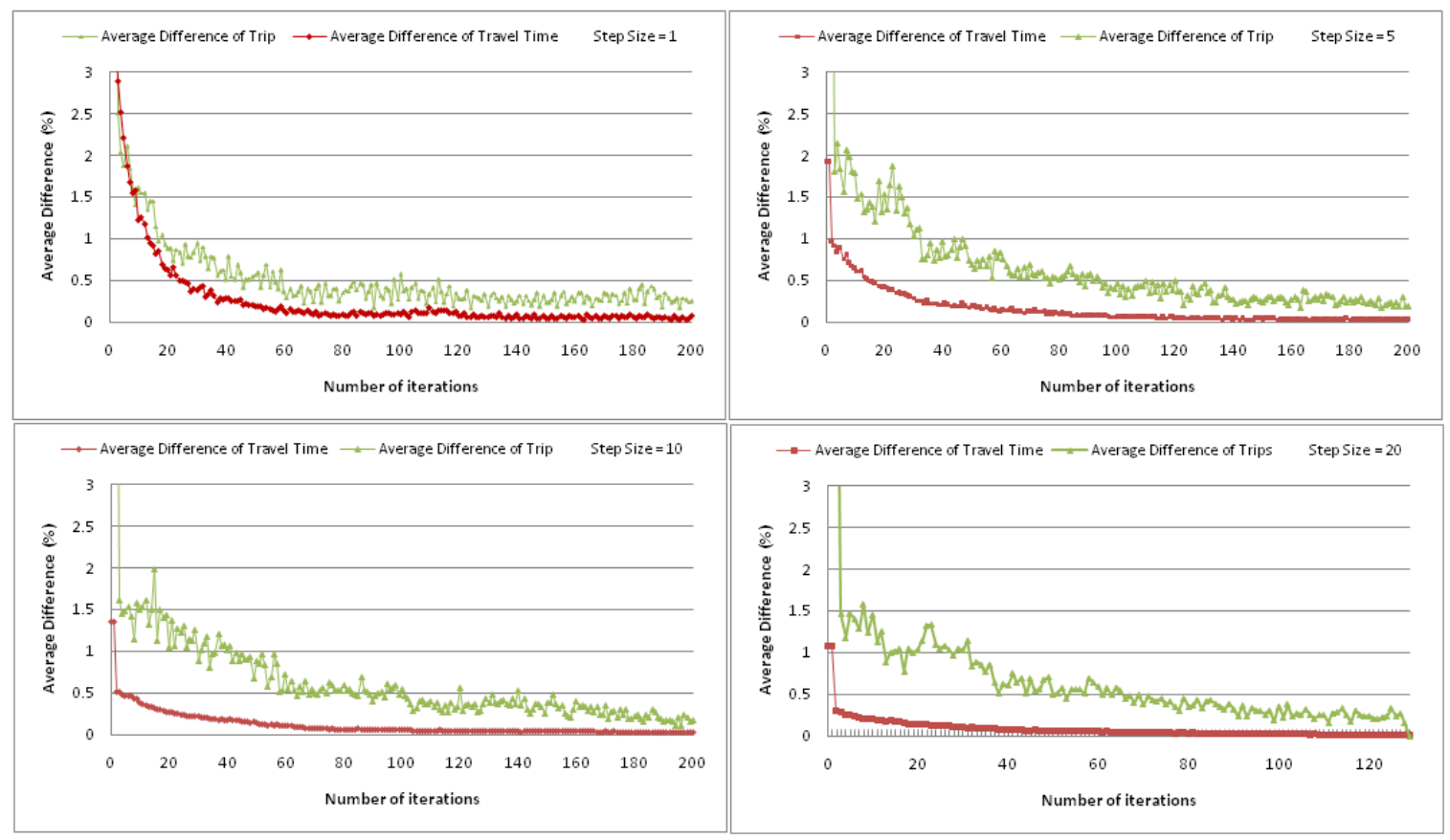

FIGURE 4: Convergence with Different Step Sizes 


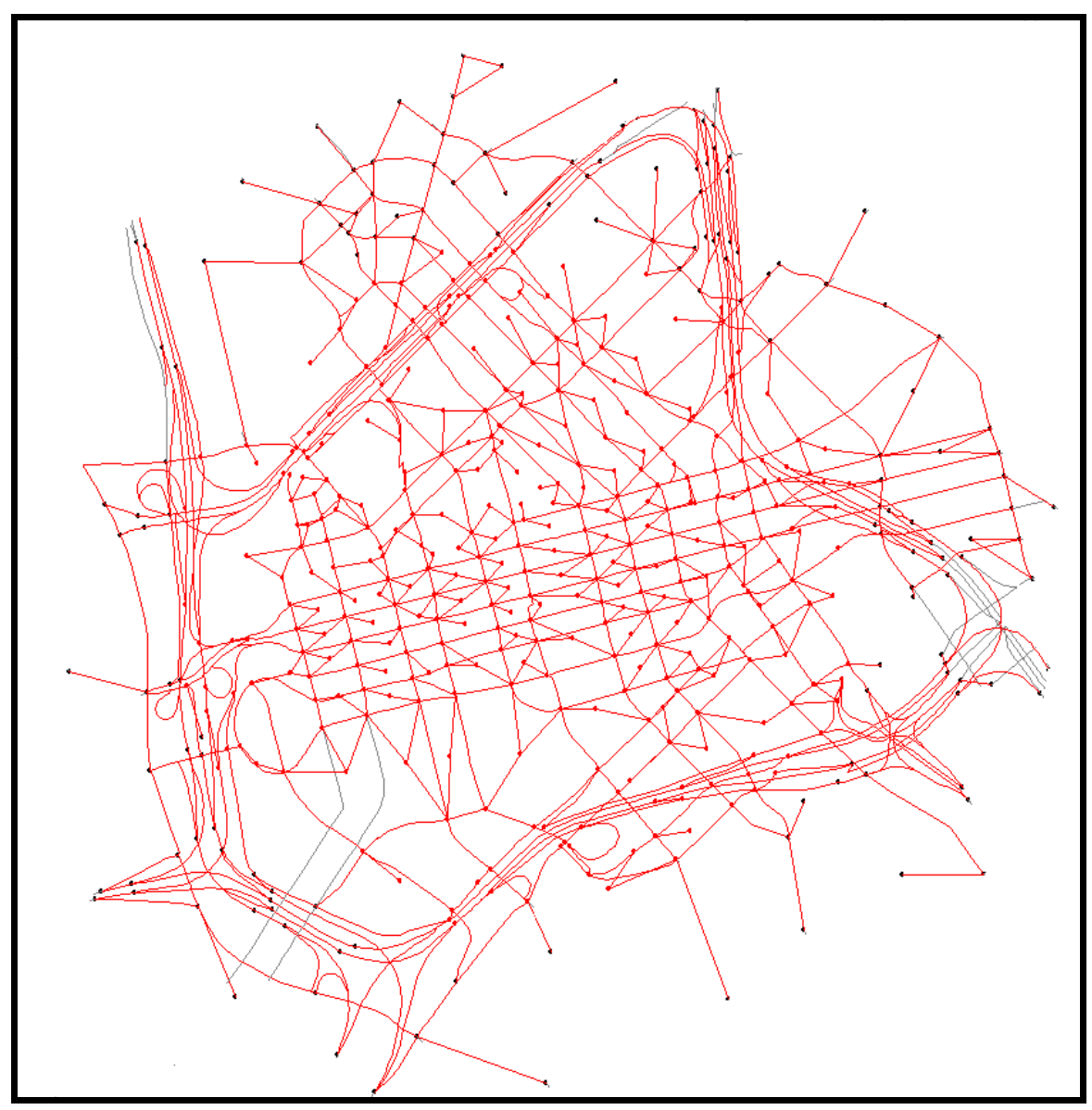

FIGURE 5: Ring Roadway Network 


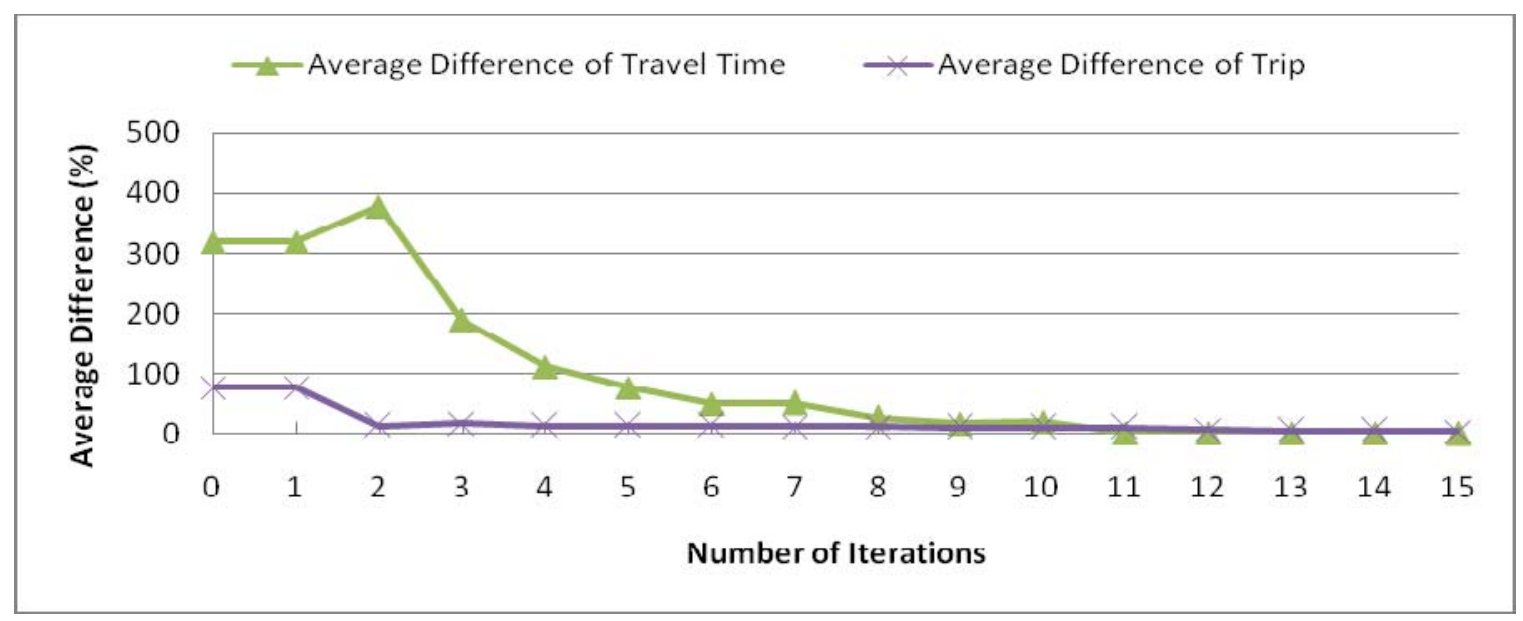

FIGURE 6: Multiple Time-intervals and Trip Table Convergence 